\title{
Phase equilibria in the polydisperse Zwanzig model of hard rods
}

\author{
Nigel Clarke ${ }^{1}$, José A. Cuesta ${ }^{2}$, Richard Sear ${ }^{3}$, Peter Sollich ${ }^{4}$, Alessandro Speranza ${ }^{4}$ \\ ${ }^{1}$ Materials Science Centre, UMIST and The University of Manchester, \\ Manchester M1 7HS, U.K.; nigel.clarke@umist.ac.uk \\ ${ }^{2}$ GISC, Dpto. de Matemáticas, Universidad Carlos III de Madrid, Avda. de la Universidad 30 \\ 28911 - Leganés, Madrid, Spain; cuesta@math.uc3m.es \\ ${ }^{3}$ Department of Physics, University of Surrey, Guildford, \\ Surrey GU2 7XH, U.K.; R.Sear@surrey.ac.uk \\ ${ }^{4}$ Department of Mathematics, King's College London, Strand, \\ London WC2R 2LS, U.K.; \{peter.sollich, alessandro.speranza\}@kcl.ac.uk
}

\begin{abstract}
We study the phase behaviour of the Zwanzig model of suspensions of hard rods, allowing for polydispersity in the lengths of the rods. In spite of the simplified nature of the model (rods are restricted to lie along one of three orthogonal axes), the results agree qualitatively with experimental observations: the coexistence region broadens significantly as the polydispersity increases, and strong fractionation occurs, with long rods found preferentially in the nematic phase. These conclusions are obtained from an analysis of the exact phase equilibrium equations. In the second part of the paper, we consider the application of the recently developed "moment free energy method" to the polydisperse Zwanzig model. Even though the model contains non-conserved densities due to the orientational degrees of freedom, most of the exactness statements (regarding the onset of phase coexistence, spinodals, and critical points) derived previously for systems with conserved densities remain valid. The accuracy of the results from the moment free energy increases as more and more additional moments are retained in the description. We show how this increase in accuracy can be monitored without relying on knowledge of the exact results, and discuss an adaptive technique for choosing the extra moments optimally.
\end{abstract}

\section{INTRODUCTION}

Solutions of rod-like particles undergo a transition from a disordered isotropic phase to an ordered nematic phase as the concentration of rods is increased. In the isotropic phase the rods have no preferred orientation, whereas in the nematic phase there is a favoured average alignment of the rods. Such a transition has been observed by Zocher 1 in solutions of rod-like $\mathrm{V}_{2} \mathrm{O}_{5}$ particles, and later by Bawden, Pirie, Bernal and Fankuchent. 3 ip solutions of tobacco mosaic virus. In 1949, Onsager showed that the ordering could be explained, at least for solutions of monodisperse long thin rigid rods, by considering only the competition between the excluded volume interaction and the orientational entropy. The key to his model is the distribution function, $P(\theta)$, that represents the fraction of rods with a given angular orientation, $\theta$, with respect to some director. In principle, this function can be determined self-consistently by minimizing the free energy; however, the resultant non-linear integral equation for $P(\theta)$ cannot be solved analytically. To progress further, Onsager introduced a trial function with a single variational parameter, $\alpha$. By now minimizing the resultant free energy with respect to $\alpha$, its concentration dependence can be calculated. From this dependence the conditions for coexistence of the isotropic and nematic phases can be determined.

There are several difficulties in quantitatively comparing experimental results, from systems such as solutions of tobacco mosaic virus, with Onsager's predictions. Firstly, in addition to the hard core repulsion, the interaction between rods often includes a soft repulsion due to electrostatic forces. Secondly, the rods are rarely rigid, and semi-flexibility must be accounted for. To overcome these difficulties, Buining, Veldhuizen, Pathmamanoharan, Hansen and Lekkerkerker 6 synthesized sterically stabilized beohmite particles. Buining and Lekkerkerker and van Bruggen, van der Kooij and Lekkerkerker 8 then studied the phase behaviour of solutions of these particles, which can be modelled reasonably with a rigid hard rod interaction, with corrections arising from soft electrostatic repulsions and semi-flexibility being negligible. However, there remains one further complication: as with most polymeric systems, the particles are polydisperse.

The Onsager approach has been modified to enable phase diagrams for bidisperse 911 and tridisperse 12 systems, in which the rods differ only in length, to be calculated. A rich variety of behaviour has been predicted, such as widening of the region of coexistence, fractionation of the longer rods into the nematic phase, nematicnematic coexistence, and re-entrant phases. Qualitatively, the first three of these are in agreement with the experiments reported in Ref. 7 and 8, and the widening of the region of coexistence between phases appears to be a general feature of polydisperse systems. Despite the obvious difficulties in trying to map a continuous polydisperse system onto a bidisperse mixture, Merchant and Rill 13 have attempted to analyse the transition concentration in solutions of polydisperse rod-like DNA in terms of the theory presented in Ref. 10 . It is clear that quantitative agreement is still lacking. The generalisation of the Onsager approach to continuous polydispersity is, however, far from simple. The only attempts, to date, that 
we are gware of, have treated the polydispersity perturbatively 14 15; this limits the validity of the analysis to situations with rather narrow distributions of rod lengths.

Another approach to the problem of monodisperse rodlike mixtures was adopted by Zwanzig16: he restricted the orientations of the rods to be in one of three mutually perpendicular directions. This enables the exact calculation of higher order virial coefficients, and the orientational distribution can be determined without approximations. In contrast to the Onsager approach, the Zwanzig model may be readily extended to polydisperse systems. For bidisperse mixtyres it has already been shown by Clarke and McLeish 17 that the qualitative features of the phase diagram, with the exception of the nematic-nematic coexistence, are similar to those predictedby Lekkerkerker, Coulon, van der Haegen and Debliek 10 . The polydisperse Zwanzig model therefore provides a useful starting point for understanding the effects of polydispersity on the phase behaviour of hard rod systems.

A further important motivation for studying polydispersity, in this simplified model, is that it provides an interesting scenario for testing and extending the recently proposed "moment method" approach to the thermodynamic treatment of polydisperse systems 1820 . The moment method applies to systems whose excess free energy depends only on some moments of the density distribution describing a polydisperse system; we show below that the Zwanzig model (treated within the second virial approximation) is of exactly this form. By expressing the ideal part of the free energy in a similar form a "moment free energy" can be defined, which only depends on the given moment densities. This is a drastic reduction in the number of densities required to describe the system, from the infinite number of degrees of freedom of the complete density distribution to a finite number of moment densities. The standard methods of the thermodynamics of finite mixtures can then be applied to the moment free energy to analyse the phase behaviour. Although in general the results will be approximate for systems with conserved densities it has been shown 18 that the cloud and shadow points (which specify the onset of phase coexistence in polydisperse systems), spinodals, and critical points are all found exactly. In the case of the Zwanzig (or Onsager) model, however, one has both conserved (rod lengths) and non-conserved (rod orientations) degrees of freedom. We show below that the moment method can be extended to this kind of scenario, and that most of the above exactness statements carry over. We also assess the accuracy of the moment method in the region where it is not exact and discuss how it can be improved systematically by retaining additional moment densities in the description.

The paper is structured as follows. In Sec. II, we describe the polydisperse Zwanzig model, give its free energy (calculated within the second virial approximation) and derive the corresponding expressions for the chemical potentials and the osmotic pressure. These results are used in Sec. III to study the exact thermodynamic behaviour of the model. In Sec. IV, we construct the moment free energy, discuss its properties, and compare the results obtained from it with the exact ones. Because the exact calculation of the phase behaviour of the polydisperse Zwanzig model is feasible, it might seem unnecessary to confirm these results using the moment method. However, it is precisely because the exact results are available that the Zwanzig model provides a useful test case for the application of the moment method to systems with non-conserved degrees of freedom. The conclusions drawn should help us to apply the method to more complicated systems (such as the polydisperse Onsager model) where an exact calculation of the phase behaviour is infeasible. This and other possible avenues for future work are discussed in Sec. V.

\section{DEFINITION OF THE MODEL}

The Zwanzig model considers hard rods in the shape of parallelepipeds of length $L$ and with square base of edge length $D$. In contrast to the full Onsager model, the orientations of these rods are restricted to be along one of the three cartesian coordinate axes, $\mathrm{x}, \mathrm{y}$ or $\mathrm{z}$. We will assume that all rods have the same diameter $D$, but that they are polydisperse in length, so that there is a continuous distribution of rod lengths $L$. Introducing a reference length $L_{0}$ and the normalized lengths $l=L / L_{0}$, we will focus on the Onsager limit of long thin rods. This corresponds to letting $D / L_{0} \rightarrow 0$ while keeping the normalized lengths $l$ constant. Unless a distinction between normalized $(l)$ and unnormalized $(L)$ lengths needs to be made explicitly, we will simply refer to $l$ as the length of a rod in the following.

Because of the length polydispersity, the number densities of the rods in the three possible orientations are specified by density distributions $\rho_{d}(l)$, with $d=\mathrm{x}, \mathrm{y}, \mathrm{z}$; for a small range of lengths $d l, \rho_{d}(l) d l$ is the number density of rods oriented along $d$ and with lengths between $l$ and $l+d l$. The total number density distribution (irrespective of orientation) is then

$$
\rho(l)=\rho_{\mathrm{x}}(l)+\rho_{\mathrm{y}}(l)+\rho_{\mathrm{z}}(l) \equiv \sum_{d} \rho_{d}(l)
$$

and integrating over $l$ gives the total number density of rods:

$$
\rho=\int d l \rho(l)=\sum_{d} \int d l \rho_{d}(l)
$$

Here and in the following, all integrals over $l$ run from 0 to $\infty$. With these definitions, the density distribution $\rho_{d}(l)$ over lengths $l$ and orientations $d$ can be decomposed as

$$
\rho_{d}(l)=\rho(l) P_{l}(d)=\rho P(l) P_{l}(d)
$$


where

$$
P(l)=\rho(l) / \rho
$$

is the normalized distribution of rod lengths $l$, and

$$
P_{l}(d)=\frac{\rho_{d}(l)}{\rho(l)}
$$

is the probability of finding a rod with given length $l$ in orientation $d$. Note that $P_{l}(d)$ is the analogue of the orientation distribution in the full Onsager model and obeys the normalization

$$
\sum_{d} P_{l}(d)=1
$$

In the isotropic phase, $P_{l}(d)=1 / 3$ for all $d$ and $l$. In the nematic phase, on the other hand, we have $P_{l}(\mathrm{x})=$ $P_{l}(\mathrm{y})<P_{l}(\mathrm{z})$ if we take the director to be along the $z$-axis. We will nevertheless develop the theory of the model first for arbitrary orientation distributions (with $\left.P_{l}(\mathrm{x}) \neq P_{l}(\mathrm{y})\right)$ because this leads to somewhat more compact expressions, and only specialize to the nematic case at a later stage.

For simplicity, we only treat the model in the second virial approximation. (In contrast to the case of the Onsager model, this approximation does not become exact in the limit $D L_{0} \rightarrow 0$ here: higher order virial terms to not vanish 16.) The excess free energy is then essentially determined by the excluded volume of two rods. If the rods have (normalized) lengths $l$ and $l^{\prime}$ and are perpendicular, this volume is

$$
V_{\perp}^{\text {excl }}=2 D(L+D)\left(L^{\prime}+D\right)=2 l l^{\prime}\left(D L_{0}^{2}\right)\left[1+\mathcal{O}\left(D / L_{0}\right)\right]
$$

while the excluded volume for parallel rods

$V_{\|}^{\text {excl }}=4 D^{2}\left(L+L^{\prime}\right)=4\left(l+l^{\prime}\right)\left(D L_{0}^{2}\right) \frac{D}{L_{0}}=D L_{0}^{2} \mathcal{O}\left(D / L_{0}\right)$

is negligible by comparison in the limit $D / L_{0} \rightarrow 0$. As suggested by the result for $V_{\perp}^{\text {excl }}$, we choose $D L_{0}^{2}$ as our unit of volume in the following, making all densities dimensionless $\left(\rho \rightarrow \rho D L_{0}^{2}\right)$. If we also set $k_{\mathrm{B}} T=1$, the excess free energy density becomes (within the second virial approximation)

$$
\begin{aligned}
\tilde{f} & =2 \int d l d l^{\prime} l l^{\prime}\left[\rho_{\mathrm{x}}(l) \rho_{\mathrm{y}}\left(l^{\prime}\right)+\rho_{\mathrm{x}}(l) \rho_{\mathrm{z}}\left(l^{\prime}\right)+\rho_{\mathrm{y}}(l) \rho_{\mathrm{z}}\left(l^{\prime}\right)\right] \\
& =2\left(\phi_{\mathrm{x}} \phi_{\mathrm{y}}+\phi_{\mathrm{x}} \phi_{\mathrm{z}}+\phi_{\mathrm{y}} \phi_{\mathrm{z}}\right) \\
& =\sum_{d} \phi_{d}\left(\phi-\phi_{d}\right)
\end{aligned}
$$

where we have defined

$$
\begin{aligned}
\phi_{d} & =\int d l \rho_{d}(l)=\int d l l \rho(l) P_{l}(d) \\
\phi & =\sum_{d} \phi_{d}=\int d l l \rho(l)
\end{aligned}
$$

Our choice of volume units implies that $\left(D / L_{0}\right) \phi_{d}$ and $\left(D / L_{0}\right) \phi$ are, respectively, the volume fraction of rods pointing in direction $d$ and the total volume fraction. The ratio

$$
m=\phi / \rho=\int d l l P(l)
$$

is then simply the average length of rods in the system, i.e., the first moment of the rod length distribution $P(l)$.

Adding the ideal part of the free energy (density) to Eq. (雨), we have for the total free energy (density)

$$
f=\sum_{d} \int d l \rho_{d}(l)\left[\ln \rho_{d}(l)-1\right]+\sum_{d} \phi_{d}\left(\phi-\phi_{d}\right)
$$

This equation is the starting point of our analysis. When we refer to "exact" results in the following, we mean the exact thermodynamics of the model defined by the free energy (6).

Eq. (6) shows that the free energy is a functional of the density distribution $\rho_{d}(l)$ over $l$ and $d$. If we use Eq. (2) to write $\rho_{d}(l)=\rho(l) P_{l}(d)$, we note an important difference between the two factors: while the total density distribution $\rho(l)$ is conserved (because the rods cannot change length), the orientation distribution $P_{l}(d)$ is not (because the rods can change orientation). We separate out the respective contributions to the free energy by writing

$$
\begin{aligned}
f= & \int d l \rho(l)[\ln \rho(l)-1]+\int d l \rho(l) \sum_{d} P_{l}(d) \ln P_{l}(d) \\
& +\sum_{d} \phi_{d}\left(\phi-\phi_{d}\right)
\end{aligned}
$$

For a given $\rho(l)$, the orientation distributions $P_{l}(d)$ (for each $l$ ) are then obtained by minimizing $f$, subject to the normalization constraints (3) (again, for each $l$ ). Introducing Lagrange multipliers $\kappa(l)$ for these constraints gives the minimization condition

$$
\begin{aligned}
& \frac{\delta}{\delta P_{l}(d)}\left(f+\int d l \kappa(l) \sum_{d} P_{l}(d)\right)= \\
& \rho(l)\left[\ln P_{l}(d)+1\right]+2\left(\phi-\phi_{d}\right) l \rho(l)+\kappa(l)=0
\end{aligned}
$$

Solving for $P_{l}(d)$ and eliminating the $\kappa(l)$ by using Eq. (3) gives the orientation distributions

$$
P_{l}(d)=\frac{e^{2\left(\phi_{d}-\phi\right) l}}{\sum_{d^{\prime}} e^{2\left(\phi_{d^{\prime}}-\phi\right) l}}=\frac{e^{2 \phi_{d} l}}{\sum_{d^{\prime}} e^{2 \phi_{d^{\prime}} l}}
$$

Inserting this result into the definition (5a) then gives three simultaneous nonlinear equations which can be solved for the $\phi_{d}$.

To derive the conditions for phase coexistence in the polydisperse Zwanzig model, we need expressions for the chemical potential $\mu(l)$ - which, due to the polydispersity, is a function of the rod length $l$-and the osmotic pressure. The chemical potential 


$$
\mu(l)=\frac{\delta f}{\delta \rho(l)}
$$

is obtained by functional differentiation of the free energy (7) w.r.t. $\rho(l)$. There is no contribution from the variation of $P_{l}(d)$ with $\rho(l)$ because we have already minimized the free energy w.r.t. $P_{l}(d)$. This leads to

$$
\mu(l)=\ln \rho(l)+\sum_{d} P_{l}(d) \ln P_{l}(d)+2 \sum_{d}\left(\phi-\phi_{d}\right) P_{l}(d) l
$$

or, using Eq. (8),

$$
\mu(l)=\ln \rho(l)-\ln \left(\sum_{d} e^{2\left(\phi_{d}-\phi\right) l}\right)
$$

The osmotic pressure can be written in terms of the free energy and the chemical potential; hence, using Eq. (9),

$$
\Pi=-f+\int d l \rho(l) \mu(l)=\rho+\sum_{d} \phi_{d}\left(\phi-\phi_{d}\right)
$$

\section{EXACT PHASE COEXISTENCE CALCULATION}

\section{A. Coexistence conditions}

We can now state the conditions for coexistence of two or more phases, labelled by $a=1 \ldots K$, into which a "parent" phase with density distribution $\rho^{(0)}(l)$ is assumed to have split. From Eq. (10), the equality of the chemical potentials between the phases is obeyed exactly if the densities can be written in the form

$$
\rho^{(a)}(l)=R(l) \sum_{d} \exp \left[\alpha_{d}^{(a)} l\right]
$$

with a function $R(l)$ common to all phases and the $\alpha_{d}^{(a)}$ obeying

$$
\alpha_{d}^{(a)}=2\left(\phi_{d}^{(a)}-\phi^{(a)}\right)+c
$$

Here $c$ is an arbitrary constant (again common to all phases). If the phases occupy fractions $v^{(a)}$ of the total system volume, particle conservation implies

$$
\sum_{a} v^{(a)} \rho^{(a)}(l)=\rho^{(0)}(l)
$$

This fixes $R(l)$, giving

$$
\rho^{(a)}(l)=\rho^{(0)}(l) \frac{\sum_{d} \exp \left[\alpha_{d}^{(a)} l\right]}{\sum_{a^{\prime}} v^{\left(a^{\prime}\right)} \sum_{d^{\prime}} \exp \left[\alpha_{d^{\prime}}^{\left(a^{\prime}\right)} l\right]}
$$

The density distributions over rod lengths $l$ and orientations $d$ are then found from Eq. (2) and, using Eqs. (8, 13), take the simple form

$$
\rho_{d}^{(a)}(l)=\rho^{(0)}(l) \frac{\exp \left[\alpha_{d}^{(a)} l\right]}{\sum_{a^{\prime}} v^{\left(a^{\prime}\right)} \sum_{d^{\prime}} \exp \left[\alpha_{d^{\prime}}^{\left(a^{\prime}\right)}\right]}
$$

Integrals over these distributions define, by Eqs. (1 1 .5), the values of the densities $\rho^{(a)}$ and volume fractions $\phi_{d}^{(a)}$, $\phi^{(a)}$ in all phases. These variables determine the pressures

$$
\Pi^{(a)}=\rho^{(a)}+\sum_{d} \phi_{d}^{(a)}\left(\phi^{(a)}-\phi_{d}^{(a)}\right)
$$

in the different phases; at phase coexistence, these must of course all be equal. We thus have, in the most general form of the conditions for coexistence of $K$ phases, $4 K$ variables (three $\alpha_{d}^{(a)}$ and one $v^{(a)}$ per phase $a=1 \ldots K$ ) and equally many equations to solve: the $3 K$ conditions (13) for chemical potential equality, the $K-1$ conditions (16) for equality of the pressures, and the trivial normalization of the phase volume fractions, $\sum_{a} v^{(a)}=1$.

It is easy to show that, just as in the Onsager model, isotropic-isotropic coexistence is not possible in the Zwanzig model with length polydispersity only. (This would be different if the rod diameters were polydisperse as well; compare Refs. 21 24.) Given the results for the bidisperse case 27 , it is also unlikely that nematic-nematic coexistence could occur; this is in-fontrast to what has been found for the Onsager model 10 . Intuitively, the difference can be explained as follows: when a polydisperse nematic phase splits into two nematics containing predominantly short and long rods, respectively, it gives up entropy of mixing but gains orientational entropy. In the Onsager model, where the rod angles are continuous variables, the gain in orientational entropy can be arbitrarily large, thus favouring such a phase split. (The orientational entropy tends to $-\infty$ as the orientational distribution function tends to a delta function.) In the Zwanzig case, on the other hand, the maximum gain in orientational entropy is $k_{\mathrm{B}} \ln 3$ (this being the difference between the entropies of an isotropic and a fully ordered nematic phase) so that nematic-nematic coexistence is disfavoured.

We therefore now specialize to coexistence between an isotropic (I) and a nematic (N) phase. If we choose the director to be along the $z$-axis, we then have $\phi_{\mathrm{x}}=\phi_{\mathrm{y}}$ and $\phi=2 \phi_{\mathrm{x}}+\phi_{\mathrm{z}}$. Denoting

$$
\Delta=\phi_{\mathrm{z}}-\phi_{\mathrm{x}}=\phi_{\mathrm{z}}-\phi_{\mathrm{y}}
$$

the volume fractions of rods with the three possible orientations can be expressed as

$$
\phi_{\mathrm{x}}=\phi_{\mathrm{y}}=\frac{1}{3}(\phi-\Delta), \quad \phi_{\mathrm{z}}=\frac{1}{3}(\phi+2 \Delta)
$$


and the excess free energy and pressure simplify to

$$
\begin{aligned}
\tilde{f} & =\frac{2}{3}\left(\phi^{2}-\Delta^{2}\right) \\
\Pi & =\rho+\frac{2}{3}\left(\phi^{2}-\Delta^{2}\right)
\end{aligned}
$$

Instead of numbering the phases by $a=1,2$, we label them with superscripts $\mathrm{I}$ and $\mathrm{N}$ from now on. In the isotropic phase, we have $\phi_{d}^{\mathrm{I}}=\phi^{\mathrm{I}} / 3$ for $d=\mathrm{x}, \mathrm{y}, \mathrm{z}\left(\Delta^{\mathrm{I}}=0\right)$, and by choosing the arbitary constant $c$ in Eq. (13) as $c=$ $4 \phi^{\mathrm{I}} / 3$ we can ensure that all the coefficients $\alpha_{d}^{\mathrm{I}}$ vanish. If, for the corresponding coefficients in the nematic phase, we write

$$
\alpha_{\mathrm{x}}^{\mathrm{N}}=\alpha_{\mathrm{y}}^{\mathrm{N}} \equiv \alpha_{\perp}, \quad \alpha_{\mathrm{z}}^{\mathrm{N}} \equiv \alpha_{\|}
$$

the conditions (13) simplify to

$$
\begin{aligned}
\alpha_{\|} & =\frac{4}{3}\left(\phi^{\mathrm{I}}-\phi^{\mathrm{N}}+\Delta\right) \\
\alpha_{\perp} & =\frac{4}{3}\left(\phi^{\mathrm{I}}-\phi^{\mathrm{N}}-\frac{1}{2} \Delta\right)
\end{aligned}
$$

The condition (16) of equality of the pressures, on the other hand, becomes

$$
\rho^{\mathrm{I}}+\frac{2}{3}\left(\phi^{\mathrm{I}}\right)^{2}=\rho^{\mathrm{N}}+\frac{2}{3}\left[\left(\phi^{\mathrm{N}}\right)^{2}-\Delta^{2}\right]
$$

Note that we have dropped the subscript ' $\mathrm{N}$ ' on $\alpha_{\|}$, $\alpha_{\perp}$ and $\Delta$ because the corresponding quantities in the isotropic phase are all zero.

If we denote $v^{\mathrm{I}}=\gamma$ and $v^{\mathrm{N}}=1-\gamma$ (so that $\gamma$ is the fraction of the system volume occupied by the isotropic phase), our phase coexistence problem now takes the form of three nonlinear equations $(20,21,22)$ for $\alpha_{\|}, \alpha_{\perp}$ and $\gamma$. The densities and volume fractions appearing in these equations can be found by specializing Eqs. (1, to the case of I-N coexistence:

$$
\begin{aligned}
\rho^{\mathrm{I}} & =\int d l \rho^{\mathrm{I}}(l) \\
\phi^{\mathrm{I}} & =\int d l l \rho^{\mathrm{I}}(l) \\
\rho^{\mathrm{N}} & =\int d l \rho^{\mathrm{N}}(l) \\
\phi^{\mathrm{N}} & =\int d l l \rho^{\mathrm{N}}(l) \\
\Delta & =\int d l l\left[\rho_{\|}^{\mathrm{N}}(l)-\rho_{\perp}^{\mathrm{N}}(l)\right]
\end{aligned}
$$

with

$$
\begin{aligned}
\rho^{\mathrm{I}}(l) & =\frac{3 \rho^{(0)}(l)}{3 \gamma+(1-\gamma)\left[e^{\alpha_{\|} l}+2 e^{\alpha_{\perp} l}\right]} \\
\rho_{\|}^{\mathrm{N}}(l) & =\frac{1}{3} \rho^{\mathrm{I}}(l) e^{\alpha_{\|} l} \\
\rho_{\perp}^{\mathrm{N}}(l) & =\frac{1}{3} \rho^{\mathrm{I}}(l) e^{\alpha_{\perp} l} \\
\rho^{\mathrm{N}}(l) & =\rho_{\|}^{\mathrm{N}}(l)+2 \rho_{\perp}^{\mathrm{N}}(l)
\end{aligned}
$$

In the above setting of the phase coexistence problem, we specified a single parent density distribution $\rho^{(0)}(l)$. We will normally be interested in results along a socalled "dilution line", where the parent length distribution $P^{(0)}(l)=\rho^{(0)}(l) / \rho^{(0)}$ is kept fixed while the overall parent density $\rho^{(0)}$ is varied. As $\rho^{(0)}$ is increased from zero, we then expect to find a single isotropic phase first $(\gamma=1)$. At the isotropic "cloud point", an infinitesimal fraction of nematic phase will first appear; the density $\rho^{\mathrm{N}}$ of this nematic phase gives the nematic "shadow". On the other hand, starting from high density $\rho^{(0)}$ we will first see a pure nematic phase $(\gamma=0)$. On decreasing $\rho^{(0)}$, an infinitesimal amount of isotropic phase will appear at the nematic cloud point; the density of the isotropic phase at this point gives the corresponding isotropic shadow. The two cloud points delimit the coexistence region. For values of $\rho^{(0)}$ inside this region, an isotropic and a nematic phase coexist and occupy noninfinitesimal fractions of system volume, with $\gamma$ decreasing from 1 to 0 as $\rho^{(0)}$ increases.

Numerically, rather than changing $\rho^{(0)}$ and finding $\gamma$, it is easier to vary $\gamma$ between 0 and 1 and find the corresponding $\rho^{(0)}$. To implement this scheme, one only has to replace $\rho^{(0)}(l)$ in Eqs. (24) by $\rho^{(0)}(l)=\rho^{(0)} P^{(0)}(l)$ and solve Eqs. (20,21,22) for $\alpha_{\|}, \alpha_{\perp}$ and $\rho^{(0)}$. Alternatively, one can interpret $\alpha_{\|}$and $\alpha_{\perp}$ as being defined by Eqs. 20,21), and regard $\rho^{(0)}, \rho^{\mathrm{I}}, \phi^{\mathrm{I}}, \rho^{\mathrm{N}}, \phi^{\mathrm{N}}$ and $\Delta$ as the underlying variables. Eqs. (22,23) then constitute six equations for these six unknowns, which can be solved numerically; this is the approach that we adopt.

\section{B. Results: Cloud point and shadow curves}

In the following, we will restrict ourselves to the case where the rod lengths in the parent phase are distributed according to a Schulz distribution

$$
P^{(0)}(l)=\frac{(z+1)^{z+1}}{\Gamma(z+1)} l^{z} \exp [-(z+1) l]
$$

This distribution is normalized and has an average rod length of $m^{(0)}=1$. (Allowing other values of $m^{(0)}$ would not make our treatment more general since the value of $m^{(0)}$ can always be absorbed into a rescaling of the reference length $L_{0}$.) The parameter $z$ controls the shape and width of the distribution, and is taken to be nonnegative. A more intuitive measure of the width of the parent distribution is the relative standard deviation $\sigma$ (usually called the "polydispersity"), defined by

$$
\sigma^{2}=\left(\frac{1}{\left[m^{(0)}\right]^{2}} \int d l l^{2} P^{(0)}(l)\right)-1
$$

It is then easy to see that, for the Schulz distribution,

$$
\sigma=(1+z)^{-1 / 2}
$$




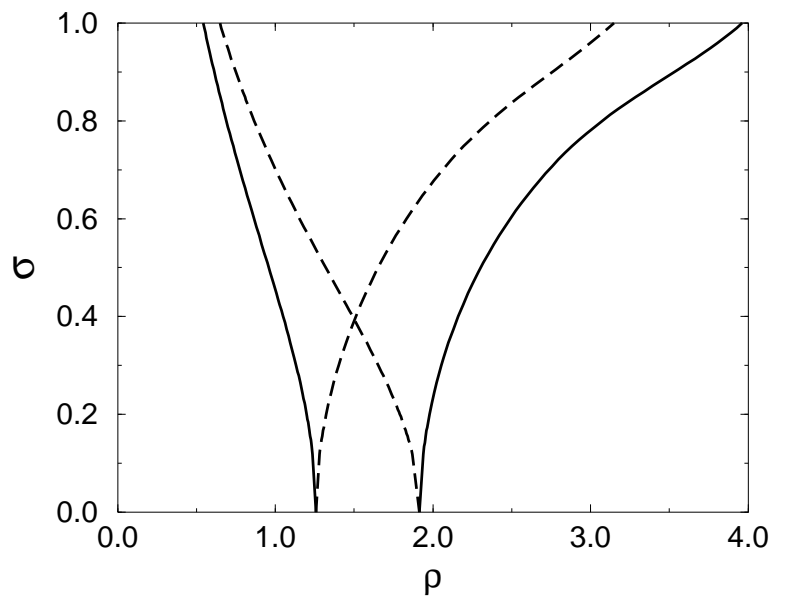

FIG. 1. The isotropic and nematic cloud point curves (solid) and their corresponding shadow curves (dashed), showing the densities of the coexisting phases as a function of the polydispersity $\sigma$. Of the two cloud point curves, the isotropic one is the one with the lower density; it meets the isotropic shadow curve for $\sigma \rightarrow 0$ as it must. The nematic cloud and shadow curves likewise coincide in this limit.

For $z \rightarrow \infty$, we thus have a monodisperse parent with $\sigma=0$ and $P^{(0)}(l)=\delta(l-1)$. As $z$ is decreased, $\sigma$ increases and the parent gets more and more polydisperse. For $z=0$, finally, the parent distribution is a simple exponential, and $\sigma$ achieves its (for the chosen Schulz distribution) maximal value of 1 .

To calculate the cloud point and shadow curves, we proceed as explained in Sec. IIIA. For the isotropic cloud point and shadow, we set $\gamma=1$. In Eqs. (24), we then have $\rho^{\mathrm{I}}(l)=\rho^{(0)}(l)$. This of course makes sense: only an infinitesimal amount of nematic phase has appeared, and so the density distribution of the isotropic phase is only negligibly perturbed away from the parent. In Eqs. (23), the equations for $\rho^{\mathrm{I}}$ and $\phi^{\mathrm{I}}$ then simplify to the trivial statements $\rho^{\mathrm{I}}=\rho^{(0)}$ and $\phi^{\mathrm{I}}=\rho^{(0)} m^{(0)}=\rho^{(0)}$, and we only have to solve four equations for the four unknowns $\rho^{(0)}, \rho^{\mathrm{N}}, \phi^{\mathrm{N}}$ and $\Delta^{\mathrm{N}}$. Conversely, for the nematic cloud point and shadow, we set $\gamma=0$. We then find $\rho^{\mathrm{N}}(l)=\rho^{(0)}(l)$ and $\rho^{\mathrm{N}}=\phi^{\mathrm{N}}=\rho^{(0)}$ and have to solve the remaining four equations for the four unknowns $\rho^{(0)}$, $\rho^{\mathrm{I}}, \phi^{\mathrm{I}}$ and $\Delta^{\mathrm{N}}$.

The results for the cloud point and shadow curves are plotted in Figs. 1 to 1 . In Fig. 1 we see that the coexistence region (the density range between the isotropic and nematic cloud points) broadens quite dramatically as the parent distribution becomes more polydisperse. The transition, which is already strongly first order in the monodisperse case, spreads out so that when $\sigma=1$ the coexistence region spans almost an order of magnitude in density, from $\rho=0.54$ to $\rho=3.96$. As $\sigma$ increases, the nematic shadow curve moves rapidly towards lower densities, approaching the isotropic cloud curve. In Fig. 2, we

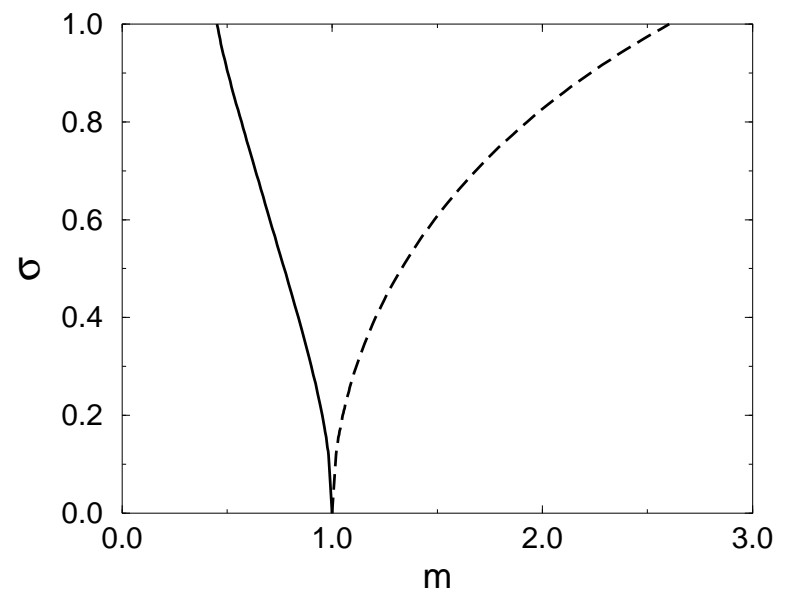

FIG. 2. The average rod lengths $m$ in the shadow phases are plotted as a function of the polydispersity, $\sigma$. The dashed and solid curves give the results for the nematic and isotropic shadow phases, respectively. Note that the corresonding cloud phases are identical to the parent and therefore have average rod lengths equal to $m^{(0)}=1$.

show the average rod lengths in the nematic and isotropic shadow phases. As the polydispersity increases, a strong fractionation effect is observed, with long rods found preferentially in the nematic phase; this is in qualitative agreement with results for the bi- and tridisperse Onsager mode 12 . For $\sigma=1$, for example, the average length of rods in the nematic phase, $m^{\mathrm{N}}$, is more than double that in the isotropic phase, $m^{\mathrm{I}}$, both at the isotropic and at the nematic cloud point. (Note that at the isotropic cloud point, $m^{\mathrm{I}}=m^{(0)}=1$ and $m^{\mathrm{N}}>1$, while at the nematic cloud point, $m^{\mathrm{N}}=m^{(0)}=1$ and $m^{\mathrm{I}}<1$.) This fractionation effect can be seen in more detail in Fig. 3, where for $\sigma=0.75$ we have plotted the relevant rod length distributions $P(l)$. For example, at $l=4$ (i.e., at four times the average rod length of the parent), $P(l)$ in the nematic shadow phase is almost an order of magnitude larger than in the isotropic cloud phase. Finally, we study in Fig. At the cloye point and shadow curves in a different representation 20: instead of the number density $\rho$ of the coexisting phases (as in Fig. 1), we show their rescaled rod volume fraction $\phi=m \rho$. This leaves the cloud point curves (for which $m=m^{(0)}=1$ ) unchanged, but does affect the shadow curves (along which, as Fig. 2 shows, $m$ can differ significantly from $m^{(0)}=1$ ). Interestingly, the volume fractions of the shadow phases turn out to depend only weakly on the polydispersity $\sigma$, in contrast to their number densities (compare Fig. 国). In fact, the volume fraction in the nematic shadow phase does not even show a definite trend in its dependence on polydispersity, being a non-monotonic function of $\sigma$.

We have not so far discussed the strength of the orientational ordering in the nematic phase. This can be 


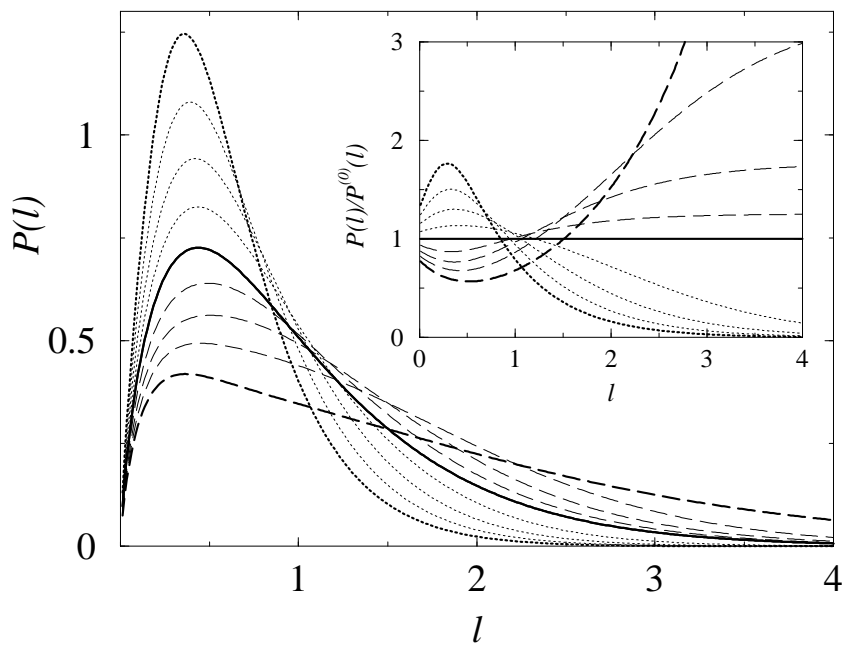

FIG. 3. The rod length distributions $P(l)$ in the coexisting isotropic and nematic phases, at polydispersity $\sigma=0.75$ and for different fractions $\gamma$ of system volume occupied by the isotropic phase. Bold dashed line: $P(l)$ in the nematic shadow at the isotropic cloud point, $\gamma=1$. The distribution in the isotropic phase at this point is the parental one (bold solid line). As $\gamma$ decreases through 0.75, 0.5, 0.25, the dashed and dotted lines show - from bottom to top - the distributions in the coexisting nematic and isotropic phases, respectively. At $\gamma=0$, finally (the nematic cloud point), the nematic has the parental length distribution; the bold dotted line shows $P(l)$ in the corresponding isotropic shadow. Inset: Ratio of the rod length distributions to that of the parent.

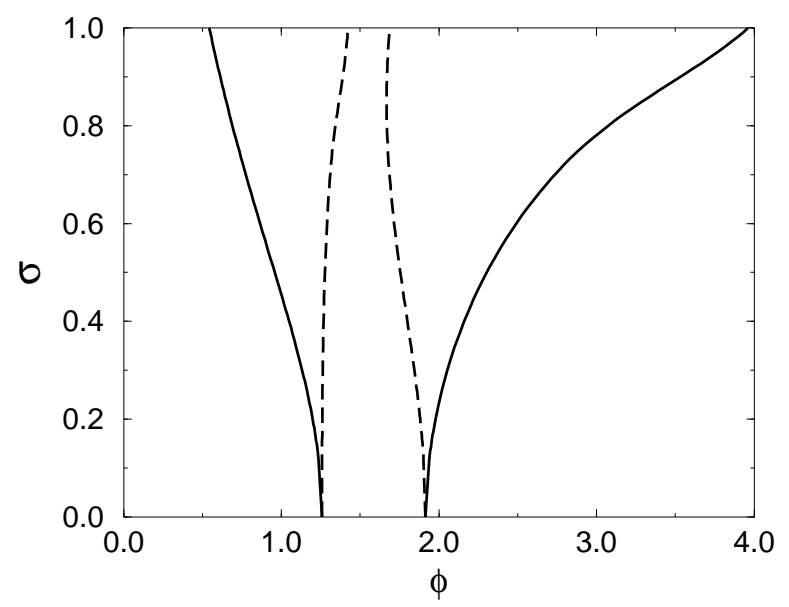

FIG. 4. The isotropic and nematic cloud point curves (solid) and their corresponding shadow curves (dashed) of Fig. 11 are replotted here, showing the (rescaled) rod volume fractions $\phi=m \rho$ of the coexisting phases rather than their densities $\rho$. Of the two cloud point curves, the isotropic one is the one with the lower $\phi$; it meets the isotropic shadow curve for $\sigma \rightarrow 0$ as it must. The nematic cloud and shadow curves likewise coincide in this limit. characterized by the order parameter $q=\Delta / \phi$, which has the value $q=0$ in the isotropic phase and $q=1$ for perfect nematic ordering. It turns out that, due to the strongly first order nature of the I-N transition, $q$ is close to its maximal value of unity for all polydispersities $\sigma$, so we do not display it.

\section{Results: Inside the coexistence region}

We now turn to the properties of the isotropic and nematic phases in the coexistence region, i.e., for parent densities between the isotropic and nematic cloud points. Both phases then exist in non-infinitesimal amounts, implying $0<\gamma<1$. Using the numerical scheme outlined in Sec. III A, we then obtain the results shown in Figs. 5 and 6. Fig. 5 tracks the densities of the coexisting phases as the coexistence region is crossed (for $\sigma=0.5$ ). As expected, the densities interpolate between the cloud and shadow phase densities at either end and increase smoothly with the parent density. Fig. 6 shows similarly the variation of the rod lengths in the isotropic and nematic phases across the coexistence region. As expected from Fig. 2, the average rod length in the nematic phase, $m^{\mathrm{N}}$, is always higher than that in the isotropic phase, $m^{\mathrm{I}}$. At the isotropic cloud point, $m^{\mathrm{I}}=1$ and $m^{\mathrm{N}}>1$, while at the nematic cloud point, $m^{\mathrm{I}}<1$ and $m^{\mathrm{N}}=1$; again the values inside the coexistence region interpolate smoothly between these limits, with both average rod lengths decreasing as the parent density $\rho^{(0)}$ increases.

Finally, we can also study the evolution of the distribution functions $P(l)$ as the fraction of volume occupied by the isotropic phase, $\gamma$, is varied. The results are included in Fig. 3. At the isotropic cloud point $(\gamma=1)$ the isotropic phase has the parental distribution $P^{(0)}(l)$; as $\gamma$ is decreased (corresponding to increasing parent density $\left.\rho^{(0)}\right)$, this distribution shifts towards smaller lengths, evolving smoothly into the distribution at the nematic cloud point $\gamma=0$. Proceeding in the reverse direction, the nematic phase has the parent distribution at $\gamma=0$ and then changes smoothly into the distribution at the isotropic cloud point as $\gamma$ is increased towards 1, shifting towards larger rod lengths in the process.

\section{COMPARISON WITH THE MOMENT METHOD}

\section{A. Constructing the moment free energy}

We now outline how the moment method 1820 can be applied to the polydisperse Zwanzig model. To construct the moment free energy, one recognizes from Eq. (18) that the excess free energy of the model (specialized to isotropic or nematic orientational order) only depends on the variables $\phi$ and $\Delta$. Both of these are moments of the 


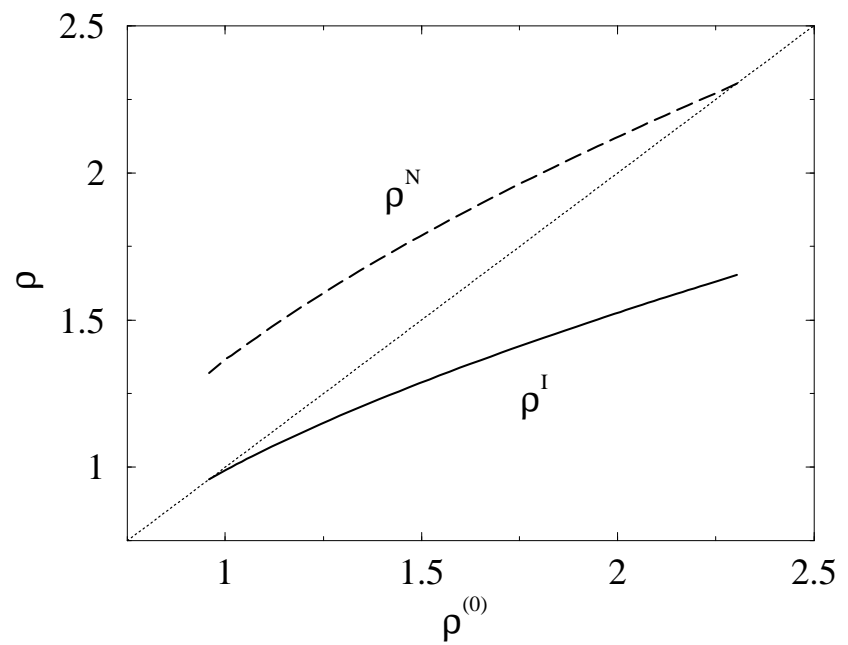

FIG. 5. The densities $\rho$ of the coexisting isotropic (solid) and nematic (dashed) phases as a function of the parent density $\rho^{(0)}$, for polydispersity $\sigma=0.5$. The isotropic and nematic cloud points, which delimit the coexistence region, are located at the densities where $\rho^{\mathrm{I}}$ and $\rho^{\mathrm{N}}$ meet the "dilution line" $\rho=\rho^{(0)}$ (dotted), respectively. Outside the coexistence region, there is only a single isotropic (for low densities) or nematic (for high densities) phase with density distribution $\rho^{(0)}(l)$ (and therefore density $\rho^{(0)}$ ) identical to that of the parent.

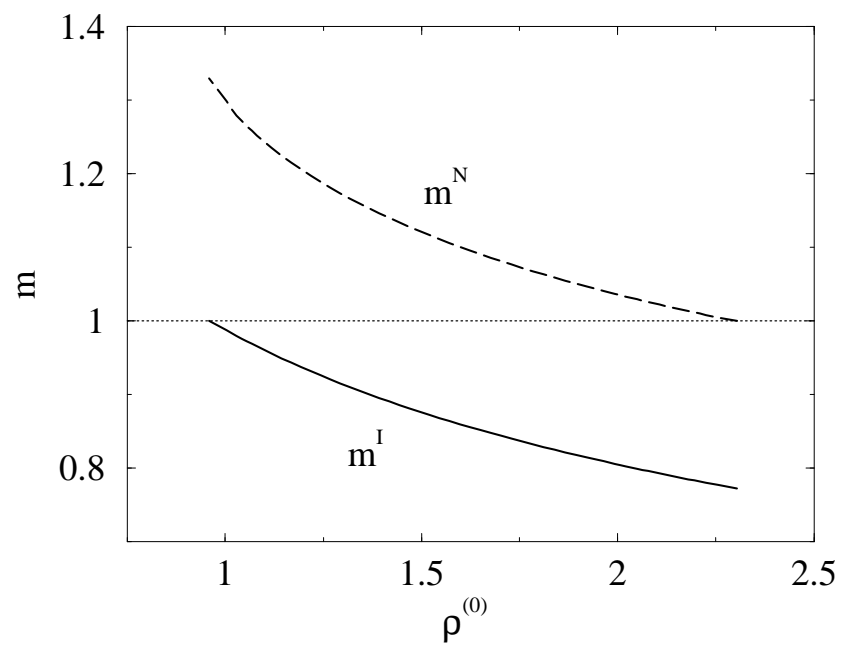

FIG. 6. The average rod lengths in the coexisting isotropic $\left(m^{\mathrm{I}}\right.$, solid) and nematic $\left(\mathrm{m}^{\mathrm{N}}\right.$, dashed) phases corresponding to Fig. 1 . The dotted line indicates the average rod length $m^{(0)}=1$ of the parent. density distribution $\rho_{d}(l)$ over lengths $l$ and orientations $d$

$$
\begin{gathered}
\phi \equiv \rho_{1}=\sum_{d} \int d l w_{1}(l, d) \rho_{d}(l) \\
\Delta \equiv \rho_{2}=\sum_{d} \int d l w_{2}(l, d) \rho_{d}(l)
\end{gathered}
$$

defined by the weight functions

$$
\begin{aligned}
& w_{1}(l, d)=l \\
& w_{2}(l, d)=l\left(\delta_{d, \mathrm{z}}-\frac{1}{2} \delta_{d, \mathrm{x}}-\frac{1}{2} \delta_{d, \mathrm{y}}\right)
\end{aligned}
$$

We therefore call $\rho_{1}$ and $\rho_{2}$ moment densities. Another, trivial, example of a moment density is the total number density

$$
\rho \equiv \rho_{0}=\sum_{d} \int d l \rho_{d}(l)
$$

which corresponds to the weight function $w_{0}(l, d)=1$.

Even though the excess free energy

$$
\tilde{f}=\frac{2}{3}\left(\rho_{1}^{2}-\rho_{2}^{2}\right)
$$

depends on moment densities only, the ideal part

$$
f_{\text {ideal }}=\sum_{d} \int d l \rho_{d}(l)\left[\ln \rho_{d}(l)-1\right]
$$

of the free energy $f=f_{\text {ideal }}+\tilde{f}$ still contains all details of the density distribution $\rho_{d}(l)$. To construct a moment free energy which depends only on the moment densities appearing in $\tilde{f}$, we therefore need to transform this ideal part to a moment form. For this purpose, it is useful to add a term $-\sum_{d} \int d l \rho_{d}(l) \ln r(l)=-\int d l \rho(l) \ln r(l)$ to the free energy, giving

$$
f=\sum_{d} \int d l \rho_{d}(l)\left[\ln \frac{\rho_{d}(l)}{r(l)}-1\right]+\tilde{f}
$$

The additional term is linear in the conserved densities $\rho(l)$ and therefore has no effect on the exact thermodynamics described by $f$. (This would not be true if we had replaced $r(l)$ by a $d$-dependent quantity $r_{d}(l)$, because $\rho_{d}(l)$ is not conserved.) For the moment method, on the other hand, $r(l)$ turns out to be crucial. The key idea is to allow violations of the particle conservation rule (14) as long as they do not affect the moment densities appearing in the excess free energy $\left(\phi \equiv \rho_{1}\right.$ and $\Delta \equiv \rho_{2}$, in our case). The intuitive rationale-to be verified a posterior - is that phase behaviour is mainly governed by the excess free energy and the moment densities appearing in it. The relevant free energy is then obtained by minimizing $f$ at given values of the moment densities $\rho_{i}(i=1,2)$. Using Lagrange multipliers $\lambda_{i}$ to 
fix these values, one finds that the minimum of $f$ occurs for density distributions of the form

$$
\rho_{d}(l)=r(l) \exp \left[\sum_{i} \lambda_{i} w_{i}(l, d)\right]
$$

and the corresponding minimum value is

$$
f_{\mathrm{mom}}=\sum_{i} \lambda_{i} \rho_{i}-\rho_{0}+\tilde{f}
$$

This expression defines the moment free energy. Note that, from Eq. (27), the moment densities are related to the Lagrange multipliers by

$$
\rho_{i}=\sum_{d} \int d l w_{i}(l, d) r(l) \exp \left[\sum_{j} \lambda_{j} w_{j}(l, d)\right]
$$

Inverting these relations determines the $\lambda_{j}$ in terms of the $\rho_{i}$; the moment free energy (28) thus depends on the moment densities only, as desired.

By construction, the moment free energy (28) is the free energy of systems with density distributions of the form (27). One therefore expects it to give exact results for the phase behaviour as long as the density distributions of all the coexisting phases are actually contained in the "family" (27). Considering an isotropic parent, with $\rho_{d}^{(0)}(l)=\rho^{(0)}(l) / 3$, we can ensure that this is true at least for the parent by choosing $r(l)=\rho^{(0)}(l) / 3$. This is the choice that we adopt from now on, giving explicitly

$$
\rho_{d}(l)=\frac{1}{3} \rho^{(0)}(l) \exp \left[\sum_{i} \lambda_{i} w_{i}(l, d)\right]
$$

for the family of density distributions and

$$
\rho_{i}=\sum_{d} \int d l w_{i}(l, d) \frac{1}{3} \rho^{(0)}(l) \exp \left[\sum_{j} \lambda_{j} w_{j}(l, d)\right]
$$

for the relation between the Lagrange multipliers $\lambda_{j}$ and the moment densities $\rho_{i}$. With this choice, the isotropic cloud point and corresponding nematic shadow will be found exactly by the moment method: at that point, the parent is only negligibly perturbed because only an infinitesimal amount of the nematic phase has appeared, while the nematic phase itself is related to the parent by exactly the kind of Gibbs-Boltzmann factor appearing in Eq. (29).

To see more formally why the moment method gives exact results for the isotropic cloud point, let us write down the resultant phase coexistence conditions and show that they are equivalent to the exact conditions (particle conservation violations apart). Associated with each of the moment densities $\rho_{i}$ is a moment chemical potential $\mu_{i}-\bar{\partial} f_{\text {mom }} / \partial \rho_{i}$. Using the Legendre transform properties 20 of $f_{\mathrm{mom}}$, one finds

$$
\begin{aligned}
& \mu_{1}=\lambda_{1}+\frac{\partial \tilde{f}}{\partial \rho_{1}}=\lambda_{1}+\frac{4}{3} \rho_{1} \\
& \mu_{2}=\lambda_{2}+\frac{\partial \tilde{f}}{\partial \rho_{2}}=\lambda_{2}-\frac{4}{3} \rho_{2}
\end{aligned}
$$

Because $\rho_{1}$ is conserved while $\rho_{2}$ is not, $\mu_{1}$ must be equal in all coexisting phases, while $\mu_{2}$ must actually be zero:

$$
\mu_{1}^{(a)}=c, \quad \mu_{2}^{(a)}=0 \quad \text { for all } a
$$

where $c$ is a constant common to all phases. To compare these conditions with the exact conditions (12,13) for equality of the chemical potentials $\mu(l)$, we write the density distributions (29) for the different rod orientations explicitly:

$$
\begin{aligned}
\rho_{\|}(l) \equiv \rho_{\mathrm{z}}(l) & =\frac{1}{3} \rho^{(0)}(l) e^{\left(\lambda_{1}+\lambda_{2}\right) l} \\
\rho_{\perp}(l) \equiv \rho_{\mathrm{x}}(l)=\rho_{\mathrm{y}}(l) & =\frac{1}{3} \rho^{(0)}(l) e^{\left(\lambda_{1}-\lambda_{2} / 2\right) l}
\end{aligned}
$$

These are of the form (12) if we identify

$$
\alpha_{\perp} \equiv \alpha_{\mathrm{x}}=\alpha_{\mathrm{y}}=\lambda_{1}-\lambda_{2} / 2, \quad \alpha_{\|} \equiv \alpha_{\mathrm{z}}=\lambda_{1}+\lambda_{2}
$$

Using Eq. (13), coexisting phases calculated from the moment free energy therefore have equal (exact) chemical potentials $\mu(l)$ if

$$
\begin{aligned}
\lambda_{1}^{(a)}+\lambda_{2}^{(a)} & =2\left(\phi_{\mathrm{z}}^{(a)}-\phi^{(a)}\right)+c \\
& =\frac{4}{3}\left(-\phi^{(a)}+\Delta^{(a)}\right)+c \\
\lambda_{1}^{(a)}-\frac{1}{2} \lambda_{2}^{(a)} & =2\left(\phi_{\mathrm{x}}^{(a)}-\phi^{(a)}\right)+c \\
& =\frac{2}{3}\left(-2 \phi^{(a)}-\Delta^{(a)}\right)+c
\end{aligned}
$$

in all phases. But from Eqs. (31) one easily sees that these conditions are equivalent to those [Eq. (32)] derived from the moment free energy, as promised. For the condition of equality of pressure in all phases, it is even easier to see that the moment free energy gives the correct answer: one finds

$$
\Pi=-f_{\mathrm{mom}}+\rho_{1} \mu_{1}+\rho_{2} \mu_{2}=\rho_{0}+\frac{2}{3}\left(\rho_{1}^{2}-\rho_{2}^{2}\right)
$$

which is exactly the same as the result (19) derived from the original free energy $f$. Remember that, in our moment density notation, $\rho_{0} \equiv \rho, \rho_{1} \equiv \phi$, and $\rho_{2} \equiv \Delta$.

We have thus shown that coexisting phases calculated from the moment free energy satisfy the exact phase equilibrium conditions of equal chemical potentials and pressures. If the phases also obey the exact particle conservation conditions (14), they therefore give the exact solution of the phase coexistence problem. This is the case at the isotropic cloud point, because one of the phases is then identical to the isotropic parent, and the other (nematic) phase is infinitesimally small. As stated above, 
this point will therefore be located exactly by the moment free energy method. The nematic cloud point, on the other hand, will not be found exactly: on the highdensity side of this point, the density distribution of the single nematic phase $\left(\rho_{d}(l)=\rho^{(0)}(l) P_{l}(d)\right.$, with $P_{l}(d)$ obeying Eq. (8)), will not in general be a member of the family 29 .

In Refs. 18,19 it was shown that the moment free energy allows one to determine exactly the onset of phase coexistence (cloud point and shadow), the spinodals and the critical points of a polydisperse system with conserved densities. In our above discussion, we have shown that for a system with non-conserved degrees of freedom (the rod orientations), the onset of phase coexistence is still located exactly under the following condition: in the single phase region from which coexistence is approached, the parent must not exhibit any ordering of the nonconserved degrees of freedoms (which means in our case that it must be isotropic). One can show that this conclusion holds quite generally, and that under the same restriction spinodals and critical points found from the moment free energy also remain exact. We thus conclude that the moment method remains useful even in systems with non-conserved degrees of freedom.

To improve the accuracy of the moment method in the regions where it is not exact (beyond the isotropic cloud point), one can simply retain additional moment densities in the moment description, defined by weight functions $w_{i}(l, d)$. The above construction of the moment free energy generalizes directly to this case: the expressions (28,29, 30) remain valid as long as the sums over $i$ are extended appropriately. From Eq. (29), one recognizes that the addition of new moment densities has the effect of extending the family of density distributions that are accessible; the exact distributions [Eq. (15)] in the coexisting phases can thus be approximated with arbitrary accuracy as the number of moment densities is increased. More explicitly, this can be seen as follows. Because the additional moment densities $\rho_{i}(i \neq 1,2)$ do not appear in the excess free energy, their associated moment chemical potentials are simply $\mu_{i}=\lambda_{i}$. These must be equal in all phases, so we can write the density distributions in coexisting phases predicted by the moment method as

$$
\begin{aligned}
\rho_{d}^{(a)}(l)= & \frac{1}{3} \rho^{(0)}(l) \exp \left[\sum_{i \neq 1,2} \lambda_{i} w_{i}(l, d)\right] \\
& \times \exp \left[\sum_{i=1,2} \lambda_{i}^{(a)} w_{i}(l, d)\right]
\end{aligned}
$$

Comparing with Eq. (12), and bearing in mind the identification (33), we see that the moment method essentially approximates $\ln (3 / D)$ (where $D$ is the denominator on the r.h.s. of Eq. (15)) by a linear combination of the additional weight functions. Since $D$ depends on $l$ only (not on $d$ ), all these weight functions can be chosen to be $d$-independent; the corresponding moment densities are thus conserved. A particular additional moment density that we will always retain is the overall density $\rho_{0}$, with weight function $w_{0}(l)=1$. This guarantees that the dilution line $\rho(l)=$ const $\times \rho^{(0)}(l)=e^{\lambda_{0}} \rho^{(0)}(l)$ for the parent $\rho^{(0)}(l)$ is contained in the family 27 , and thus simplifies the calculation of cloud points and shadows. The optimal choice of the remaining additional weight functions $w_{i}(l)$ $(i \geq 3)$ is less clear cut and is discussed further below. One thing we can say already at this point, however, concerns the large $l$ asymptotics: it is easy to see that, for large $l, \ln (3 / D)=c_{1}+c_{2} l+e^{-c_{3} l}$, with constants $c_{1}, c_{2}$, $c_{3}$, and up to terms which are exponentially smaller. The first two contributions are covered by by the weight functions $w_{0}, w_{1}, w_{2}$, so all other weight functions should be chosen to decay exponentially for large $l$; the coefficient $c_{3}$ of this decay is not known a priori, however.

\section{B. Results}

We show in Fig. 7 the cloud point and shadow curves obtained from the moment free energy with different numbers $n$ of moment densities retained. As explained above we always keep, beyond the "essential" moment densities $\rho_{1}$ and $\rho_{2}$, the overall number density $\rho_{0}$, so the smallest value of $n$ that we consider is $n=3$. For larger $n$, the additional weight functions were chosen to be exponentials with increasing decay constants, $w_{2+j}(l)=e^{-c j l}$ $(j=1 \ldots n-3)$. This form is consistent with the expected exponential behaviour for large $l$; the coefficient $c$ was chosen as $c=0.5$ by trial and error. As expected, the isotropic cloud point curve and the corresponding nematic shadow are found exactly already for $n=3$. For the nematic cloud point curve and the isotropic shadow, deviations from the exact results become apparent for larger polydispersities $\sigma$; as expected, these deviations decrease as $n$, the number of moment densities retained, increases.

As noted in the introduction, for the polydisperse Zwanzig model that we are considering the moment method is not really needed because the exact phase coexistence equations can be solved directly. However, for more realistic models (such as the polydisperse Onsager model, with unrestricted rod orientations), this will not be the case. One thus needs to be able to assess the accuracy of the moment method without knowing the exact results beforehand. In Ref. 20, the following quantity was proposed for this purpose: for any phase coexistence calculated from the moment free energy, one can work out the total density distribution over rod lengths, $\rho_{\text {tot }}(l)=\sum_{a} v^{(a)} \rho^{(a)}(l)=\sum_{a, d} v^{(a)} \rho_{d}^{(a)}(l)$. The quantity $\ln \rho_{\text {tot }}(l) / \rho^{(0)}(l)$ then measures how strongly particle conservation of rods of length $l$ is violated; taking the square and averaging over the normalized parent distribution $P^{(0)}(l)=\rho^{(0)}(l) / \rho^{(0)}$ defines the "log-error" 


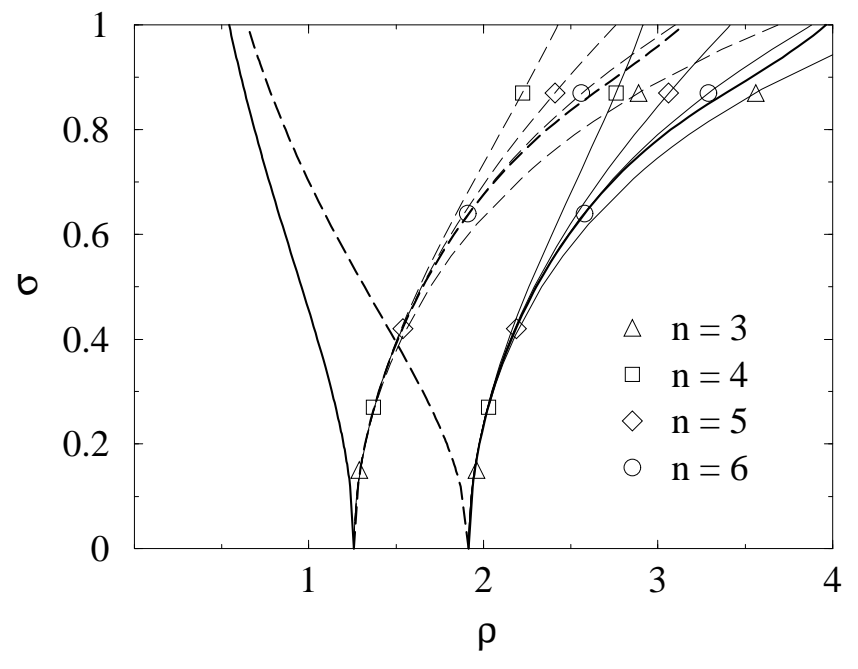

FIG. 7. Cloud point and shadow curves found using the moment method. The exact results (compare Fig. 1) are shown in bold for comparison. Even when only the minimal number of moment densities $(n=3)$ is retained in the moment free energy, the isotropic cloud point and corresponding nematic shadow are found exactly; the moment method results therefore overlay the corresponding exact curves. The nematic cloud point and isotropic shadow are not found exactly, but their accuracy increases as $n$ (indicated by the upper row of symbols) increases. The other symbols show the points on the moment method curves where the log-error $\delta$ first reaches the value $10^{-4}$ as $\sigma$ is increased from zero; see text for discussion.

$$
\delta=\int d l P^{(0)}(l)\left(\ln \frac{\rho_{\mathrm{tot}}(l)}{\rho^{(0)}(l)}\right)^{2}
$$

For small violations of particle conservation, $\ln \rho_{\text {tot }}(l) / \rho^{(0)}(l) \approx \rho_{\text {tot }}(l) / \rho^{(0)}(l)-1$, and we can think of $\sqrt{\delta}$ as the root-mean-squared relative deviation between $\rho_{\text {tot }}(l)$ and $\rho^{(0)}(l)$. In Fig. [7, we indicate by the lower symbols on the nematic cloud point and isotropic shadow curves where, as the polydispersity $\sigma$ is increased from zero, $\delta$ first reaches the value $10^{-4}$ (which corresponds to an average violation of particle conservation of $1 \%$ ). The fact that the symbols lie essentially on the curves with the exact result shows that $\delta$ provides a food indicator of the accuracy of the moment method20: adding moment densities until $\delta \leq 10^{-4}$ ensures that the results are essentially indistinguishable from the exact ones.

Beyond the calculation of phase boundaries, one would also like the moment method to give reliable results for the properties of coexisting phases inside the coexistence region. In Fig. 8, we therefore show the analogue of Fig. 5 for the moment method: the densities of the coexisting isotropic and nematic phases in the coexistence region, as a function of the parent density $\rho^{(0)}$. Again, exact results are obtained only at the isotropic cloud point; but as the number of moment densities, $n$, is increased, the

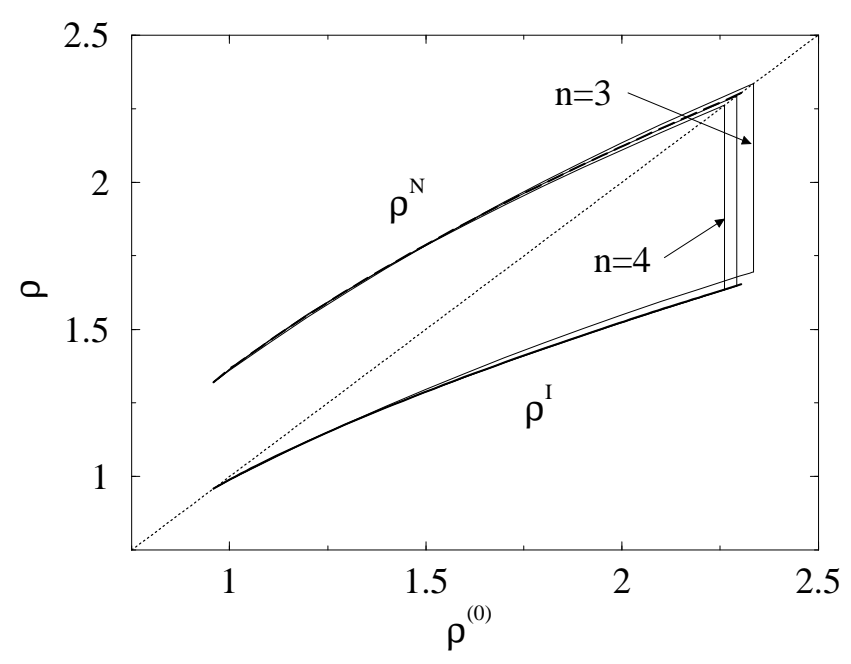

FIG. 8. The densities $\rho$ of the coexisting isotropic (solid) and nematic (dashed) phases as a function of the parent density $\rho^{(0)}$, for polydispersity $\sigma=0.5$. We show here the results calculated from the moment method with $n=3,4,5$ (thin lines), and the exact results of Fig. 月 (bold lines). As expected, the densities beyond the isotropic cloud point are not exact, but become increasingly more accurate as $n$ is increased. The results for $n=5$ are indistinguishable from the exact ones on the scale of the plot.

results across the whole of the coexistence region become progressively more accurate.

We compare different choices for the additional weight functions in Fig. 9, in terms of the dependence of the log-error $\delta$ on $n$ at a point deep within the coexistence region of the phase diagram. Results for two sets of additional weight functions are shown. The first set consists of the exponential weight functions considered above. For the second set, we chose increasing powers of $l, w_{2+j}(l)=l^{j-1} e^{-c l}(j=1 \ldots n-3)$. Note the exponential factor, which ensures the required asymptotic behaviour; $c$ was again chosen as 0.5 . We call these weight functions "power-exponential".

In this example, the exponential weight functions are seen to lead to a faster decrease of $\delta$ with $n$. However, further experimentation with other choices of weight functions may well lead to even better results, and it would clearly be desirable to have a more systematic way of constructing optimal additional weight functions. The following adaptive approach is a first step in this direction (see also Ref. 20). Consider a given point in the phase diagram, characterized in the present case by the density $\rho^{(0)}$ of the parent and its polydispersity $\sigma$. Performing a moment method phase equilibrium calculation without additional weight functions, one will find a certain $\log$-lever rule violation $\ln \rho_{\text {tot }}(l) / \rho^{(0)}(l)$ (called "log ratio" for short in the following). One then expects that adding a weight function $\left(w_{3}(l)\right.$, in our case) which has the same $l$-dependence as this log-ratio should signifi- 


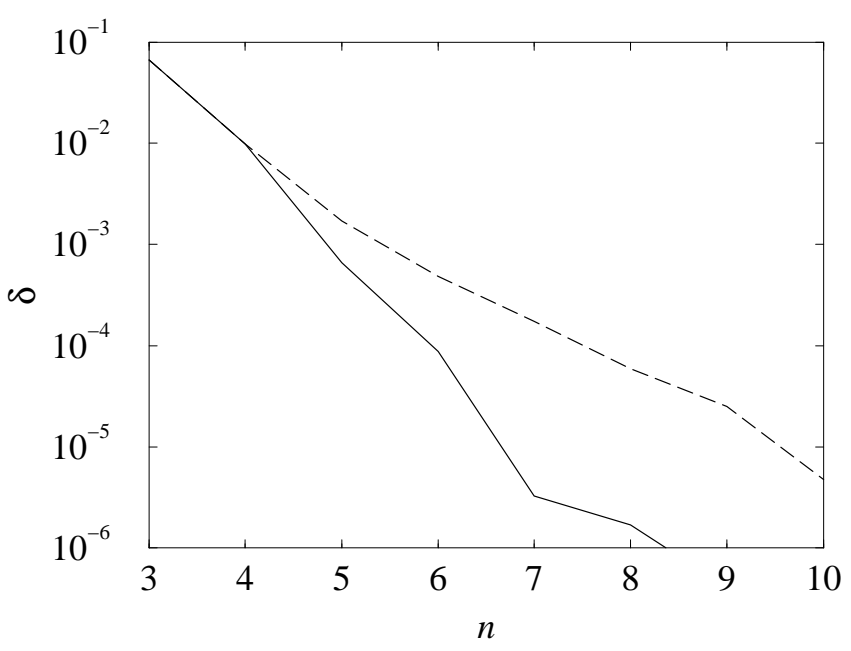

FIG. 9. The dependence of the log-error $\delta$ on the number $n$ of moment densities retained in the moment free energy, at the point $\rho^{(0)}=2, \sigma=1$ in the phase diagram. The two lines correspond to different choices of the additional weight functions: exponential (solid) and power-exponential (dashed); see text for precise functional forms.

cantly reduce the log-error: it extends the family (29) of density distributions "in the right direction". Of course, constructing $w_{3}(l)$ to fit the log-ratio exactly would be computationally costly. Instead, we represent it as a linear combination $\sum c_{k} \psi_{k}(l)$ of some simple basis functions $\psi_{k}(l)$. These could be the exponential or powerexponential functions used above, for example. The coefficients $c_{k}$ are chosen to minimize the squared deviation from the log-ratio (weighted by the normalized parent distribution),

$$
\int d l P^{(0)}(l)\left(\sum_{k} c_{k} \psi_{k}(l)-\ln \frac{\rho_{\mathrm{tot}}(l)}{\rho^{(0)}(l)}\right)^{2}
$$

This is a straightforward weighted least squares problem, and the $c_{k}$ can easily be found in closed form, thus determining $w_{3}(l)$. One can now repeat the phase equilibrium calculation with the moment $\rho_{3}$ defined by $w_{3}(l)$ included, and fit a new weight function $w_{4}(l)$ to the resulting log-ratio (which is expected to be rather smaller in magnitude than before). Repeating this process should lead to a steady decrease of the log-error $\delta$. However, a large number of additional weight functions may still be required before $\delta$ reaches an acceptably small value, and this can cause numerical problem, 25. To avoid this problem, we note from the discussion at the end of the previous section that a single additional weight function can reproduce the exact results within the moment method, if only its $l$-dependence can be found appropriately. Rather than keeping a large number of additional weight functions, we can thus continually adapt a single weight function, as follows. We choose the first additional weight function $w_{3}(l)$ by fitting the initial log-ratio, rerun the phase equilibrium calculation with $\rho_{3}$ included, and fit a "temporary" additional weight function $w_{4}(l)$ to the resulting decreased log-ratio. With both $\rho_{3}$ and $\rho_{4}$ included, we again run the calculation; this produces values of the Lagrange multipliers $\lambda_{3}$ and $\lambda_{4}$ (which, being associated with moment densities not appearing in the excess free energy, are common to all phases). The key point is now that if we merge $w_{3}(l)$ and $w_{4}(l)$ into the linear combination $w_{3}^{\prime}(l)=\lambda_{3} w_{3}(l)+\lambda_{4} w_{4}(l)$, and discard $w_{4}(l)$, repeating the calculation would give exactly the same results. (All moment phase equilibrium conditions are still satisfied, and the lever rule for $\rho_{3}^{\prime}$ obviously follows from that for $\rho_{3}$ and $\rho_{4}$.) We are now back to a situation with only a single additional weight function and can repeat the process: obtain $w_{4}^{\prime}(l)$ by fitting to the current logratio, rerun with $\rho_{3}^{\prime}$ and $\rho_{4}^{\prime}$, combine $w_{3}^{\prime}(l)$ and $w_{4}^{\prime}(l)$ into $w_{3}^{\prime \prime}(l)$ and so on. This method avoids the computational problems associated with using a large number of additional moment densities; indeed, it requires at most two additional weight functions at any time. The number of basis functions, however, is unrestricted in principle and can feasibly be made quite large. In fact, one can show that for an infinitely large set of basis functions, which allows arbitrary functional forms of the log-ratio to be fitted, the method must converge to the results of the exact phase equilibrium calculation (assuming it converges at all). For finite but sufficiently large sets of basis functions, one thus expects excellent approximations to the exact results. As long as the set of basis functions is sufficiently "flexible" to approximate the $l$-dependence of the log-ratio, the precise choice of the basis functions should also be relatively unimportant, thus reducing the effect of the remaining heuristic element of the method.

In Fig. 10, we show the results for the adaptive method just described, at the same point in the phase diagram as in Fig. 9. As basis functions we considered the exponential and power-exponential weight functions described above. The number of basis functions was chosen such that if (in the previous non-adaptive, "brute-force" approach) all basis functions are retained as additional weight functions, the log-error $\delta$ is less than $10^{-5}$. As can be read off from Fig. 9, this leads to $n-3=7-3=4$ exponential basis functions and $n-3=10-3=7$ power-exponential basis functions. The corresponding "brute-force" values of $\delta$ are shown as horizontal lines in Fig. 10. They provide natural baselines for the results of the adaptive method: because the latter only retains a single additional weight function (a linear combination of the basis functions), it can obviously do no better than the brute-force method which allows the coefficients of all basis functions to be adjusted individually. Fig. 10 confirms this; the adaptive method converges after a few iterations to a value of $\delta$ above the brute-force baseline. While the slightly larger final value of $\delta$ is, of course, a disadvantage, the adaptive method more than makes up for this by being much faster and numerically more stable. We therefore plan to study this method in more 


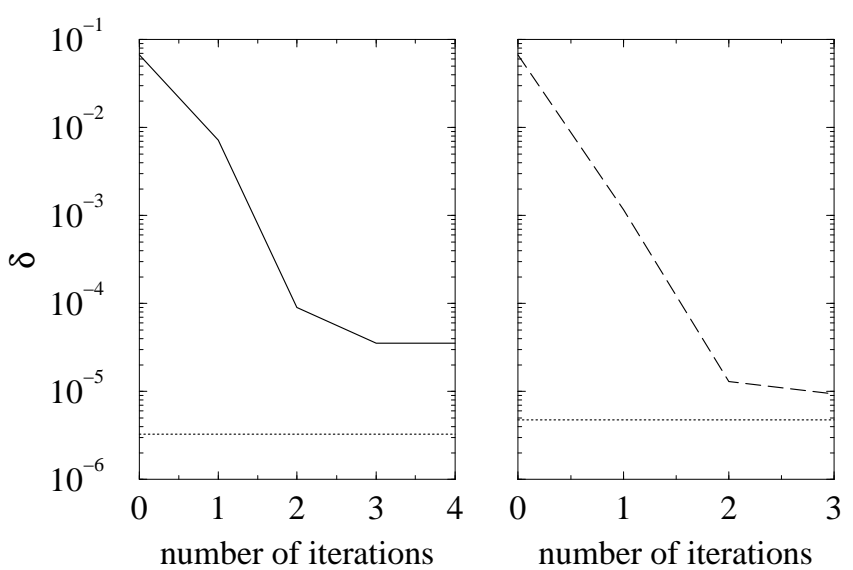

FIG. 10. The dependence of the log-error $\delta$ on the number of iterations of the "adaptive" weight function algorithm described in the text, at the point $\rho^{(0)}=2, \sigma=1$ in the phase diagram. Left: using four exponential basis functions; right: using seven power-exponential basis functions. Dotted lines: Value of $\delta$ reached by the "brute-force" approach where all basis functions are retained as weight functions.

detail in future. In particular, if one is interested in performing calculations for a number of points in the phase diagram (along a dilution line, for example, where the density $\rho^{(0)}$ of the parent is varied) one could imagine a dynamical version of the algorithm which adapts the single additional weight function whenever a threshold value of the log-error $\delta$ is crossed. As long as the chosen set of basis functions is sufficiently powerful, this should lead to uniformly precise results across the whole phase diagram.

Finally, we illustrate in Fig. 11 the geometrical intuition provided by the moment free energy. We plot $f_{\text {mom }}$ as a function of the "essential" moment densities $\rho_{1}, \rho_{2}$, for a parent $\rho^{(0)}(l)$ whose density was chosen to be exactly at the isotropic cloud point. As expected, the tangent plane drawn at the parent touches the surface at a second point: the nematic shadow phase. The moment free energy thus allows a simple geometrical interpretation of this phase transition in a polydisperse system, in terms of a double-tangent plane to a conventional two-dimensional free energy surface. We emphasize that the properties of the cloud and shadow phases are found exactly, even though the moment free energy is only a low-dimensional projection of the true free energy (which "lives" in the infinite-dimensional space of density distributions $\left.\rho_{d}(l)\right)$.

\section{CONCLUSION}

We have studied the phase behaviour of the Zwanzig model of suspensions of hard rods, allowing for polydispersity in the lengths of the rods. The model assumes

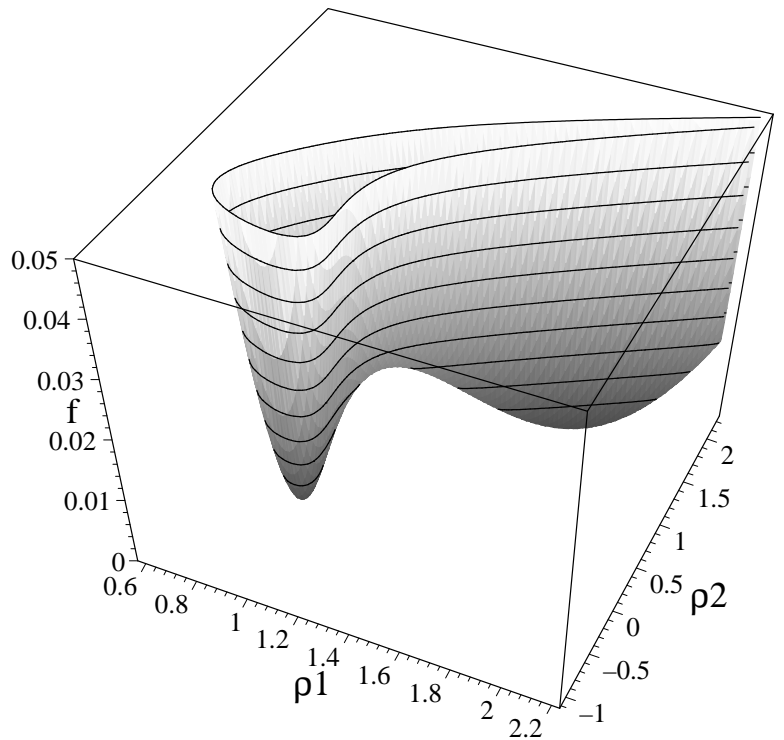

FIG. 11. Moment free energy $f_{\text {mom }}$ versus the "essential" moments densities $\rho_{1}$ and $\rho_{2}$, for a Schulz parent with $\sigma=0.5$ and density $\rho^{(0)}=0.959$ corresponding to the isotropic cloud point. Constant and linear terms have been added to $f_{\text {mom }}$ to make its tangent plane at the parent (represented by the point $\left.\rho_{1}=\rho^{(0)} m^{(0)}=0.959, \rho_{2}=0\right)$ coincide with the $x y$-plane. As expected, this tangent plane touches the free energy surface at a second point: the nematic shadow, whose values of $\rho_{1}$ and $\rho_{2}$ are found exactly.

that the rods are restricted to lie along one of three orthogonal axes. In spite of this drastic simplification (compared to the Onsager model, where rod orientations are unrestricted), the results we obtain are in qualitative agreement with experimental observations 1 6: the coexistence region broadens significantly as the polydispersity (the width of the rod length distribution of the parent) increases; fractionation is also observed, with long rods found preferentially in the nematic phase. These conclusions were obtained from an exact analysis of the phase equilibrium equations, starting from the free energy of the model within the second virial approximation.

In the second part of the paper, we considered the application of the moment method to the polydisperse Zwanzig model. This involved extending the construction of the moment free energy to models with both conserved and non-conserved degrees of freedom. We showed that most of the exactness statements obtained previously for systems with conserved densities carry over to this case: the onset of phase coexistence is still found exactly from the moment free energy, as long as it is approached from a single phase region where there is no ordering of the nonconserved degrees of freedom. With the same restriction, spinodal instabilities and critical points are also located exactly. Our concrete results for the cloud and shadow curves bear this out; the isotropic cloud point and corresponding nematic shadow are found exactly, while the 
nematic cloud point and isotropic shadow are approximate. The accuracy of the approximation increases as the number of moment densities retained in the moment free energy is increased. The log-error $\delta$ is a useful criterion for monitoring the increase in accuracy; crucially, it can be computed without knowing the exact results beforehand. Finally, we have discussed methods for choosing the weight functions of the additional moment densities. An adaptive technique, which requires at most two additional weight functions at any given time, and is therefore very cheap to implement computationally, gives promising results.

In future work, it is obviously desirable to remove the simplications of the Zwanzig model and move towards a study of the polydisperse Onsager model, with unrestricted rod orientations. A direct numerical solution of the phase equilibrium equations for this model is infeasible, so an approach based on the moment method suggests itself. One complication is that the excess free energy does not have a simple moment structure: the excluded volume between two rods at angles $\theta$ and $\theta^{\prime}$ with the nematic axis is a non-trivial function of these angles. Its expansion in terms of Legendre polynomials 26 shows that the moment free energy actually depends on an infinite number of moment densities. As an intermediate step, we therefore plan to consider the polydisperse Maier-Saupe model27, which truncates the eigenfunction expansion after the leading term and leads to an excess free energy depending on only two moment densities. This approach should be of independent interest as a phenomenological description of polydisperse suspensions of rod-like particles with more complex (soft) interactions. Finally, it would also be interesting to study the effect of diameter-polydispersity on hard rod systems. Novel features such as isotropic-isotropic phase coexistence have previously been found for the bidisperse case (rods with two different diameters)21 24, and it will be interesting to see how these are modified for truly polydisperse systems.

Acknowledgments: JC's work is part of the project PB96-0119 of the Dirección General de Enseñanza Superior. JC and RS are partly supported by project HB19980008 funded by Acciones Integradas con el Reino Unido (Ministerio de Educación y Cultura) and The British Council. Support from the EPSRC Soft Condensed Matter Network is gratefully acknowledged.

${ }^{1}$ H. Z. Zocher, Anorg. Chem. 147, 91 (1925).

${ }^{2}$ F. C. Bawden, N. W. Pirie, J. D. Bernal, and I. Fankuchen, Nature 138, 1051 (1936).

${ }^{3}$ J. D. Bernal and I. Fankuchen, J. Gen. Physiol. 25, 111 (1941).

${ }^{4}$ L. Onsager, Ann. N.Y. Acad. Sci. 51, 627 (1949).
${ }^{5}$ P. A. Buining, C. Pathmamanoharan, J. B. H. Hansen, and H. N. W. Lekkerkerker, J. Am. Ceram. Soc. 74, 1303 (1991).

${ }^{6}$ P. A. Buining, Y. S. J. Veldhuizen, C. Pathmamanoharan, and H. N. W. Lekkerkerker, Colloids Surf. 44, 47 (1992).

${ }^{7}$ P. A. Buining and H. N. W. Lekkerkerker, J. Phys. Chem. 97, 11510 (1993).

${ }^{8}$ M. P. B. van Bruggen, F. M. van der Kooij, and H. N. W. Lekkerkerker, J. Phys.: Condens. Matter 8, 9451 (1996).

${ }^{9}$ T. Odijk and H. N. W. Lekkerkerker, J. Phys. Chem. 89, 2090 (1985).

${ }^{10}$ H. N. W. Lekkerkerker, P. Coulon, R. van der Haegen, and R. Debliek, J. Chem. Phys. 80, 3427 (1984).

${ }^{11}$ G. J. Vroege and H. N. W. Lekkerkerker, J. Phys. Chem. 97, 3601 (1993).

12 G. J. Vroege and H. N. W. Lekkerkerker, Colloids Surf. A: 130, 405 (1997).

${ }^{13}$ K. Merchant and R. L. Rill, Biophys. J. 73, 3154 (1997).

${ }^{14}$ T. J. Sluckin, Liquid Crystals 6, 111 (1989).

${ }^{15}$ Z. Y. Chen, Phys. Rev. E 50, 2849 (1994).

${ }^{16}$ R. Zwanzig, J. Chem. Phys. 39, 1714 (1963).

${ }^{17}$ N. Clarke and T. C. B. McLeish, J. Phys. II (France) 2, 1841 (1992).

${ }^{18}$ P. Sollich and M. E. Cates, Phys. Rev. Lett. 80, 1365 (1998).

19 P. B. Warren, Phys. Rev. Lett. 80, 1369 (1998).

${ }^{20}$ P. Sollich, P. B. Warren, and M. E. Cates, Adv. Chem. Phys., in press.

${ }^{21}$ R. P. Sear and G. Jackson, J. Chem. Phys. 103, 8684 (1995).

22 R. P. Sear and B. M. Mulder, J. Chem. Phys. 105, 7727 (1996); 106, 3827 (1997).

${ }^{23}$ R. van Roij and B. Mulder, Phys. Rev. E 54, 6430 (1996).

${ }^{24}$ R. van Roij, B. Mulder, and M. Dijkstra, Physica A 261, 374 (1998).

${ }^{25}$ Note that once the number of additional weight functions equals the number of basis functions, the iterative process can be stopped because no further improvement is possible: the adaptively chosen weight functions then span the same space as the basis functions. All results will therefore be the same as if the basis functions themselves had been chosen as the additional weight functions, and adding further weight functions does not enlarge the family (29) of accessible density distributions.

${ }^{26}$ R. F. Kayser and H. J. Raveche, Phys. Rev. A 17, 2067 (1978).

${ }^{27}$ W. Maier and A. Saupe, Z. Naturforsch. A 13, 564 (1958). 